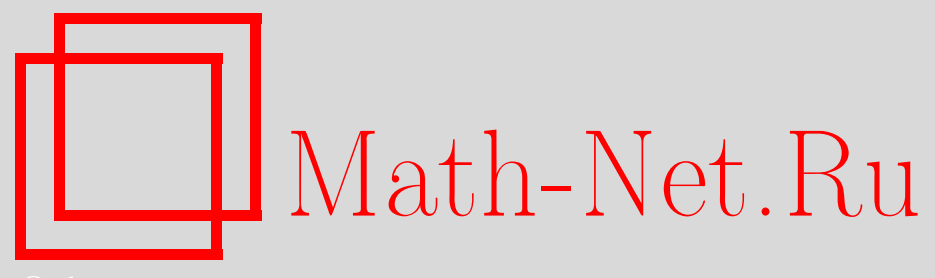

И. Э. Келлер, В. Н. Трофимов, А. В. Владыкин, В. В. Плюснин, Д. С. Петухов, И. В. Виндокуров, K вопросу о реконструкции остаточных напряжений и деформаций пластины после дробеструйной обработки, Вестн. Сам. гос. техн. ун-та. Сер. Физ.-мат. науки, 2018, номер 1, 40-64

DOI: https://doi.org/10.14498/vsgtu1602

Использование Общероссийского математического портала MathNet.Ru подразумевает, что вы прочитали и согласны с пользовательским соглашением

http://www . mathnet.ru/rus/agreement

Параметры загрузки:

IP : 35.173 .137 .237

26 апреля 2023 г., 15:35:28

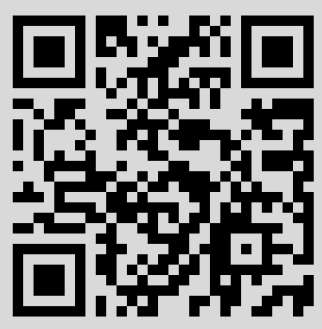


Вестн. Сам. гос. техн. ун-та. Сер. Физ.-мат. науки. 2018. Т. 22, № 1 . С. $40-64$ ISSN: 2310-7081 (online), 1991-8615 (print)

УДК 539.373

\title{
К вопросу о реконструкции остаточных напряжений и деформаций пластины после дробеструйной обработки
}

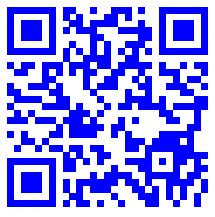

\author{
И. Э. Келлер ${ }^{1,2}$, В. Н. Трофимов ${ }^{1}$, А. В. Владыкин ${ }^{3}$, \\ В. В. Плюснин ${ }^{3}$, Д. С. Петухов ${ }^{1,2}$, И. В. Виндокуров ${ }^{1}$
}

1 Пермский национальный исследовательский политехнический университет,

Россия, 614990, Пермь, Комсомольский проспект, 29.

2 Институт механики сплошных сред УрО РАН,

Россия, 614013, Пермь, ул. Акад. Королёва, 1.

3 АО «ОДК-Пермские моторы»,

Россия, 614010, Пермь, Комсомольский проспект, 93.

\section{Аннотация}

Предметом исследования является математическое описание формы и напряженно-деформированного состояния стальной пластины, подвергнутой односторонней дробеструйной обработке, его экспериментальное подтверждение и применение результатов для верификации методов реконструкции полей остаточных напряжений и деформаций по экспериментальным данным. Подобная пластина используются на производстве в качестве калибровочного образца для определения времени пневмодробеструйной обработки, необходимого для формирования в поверхностном слое обрабатываемого изделия сжимающих тангенциальных остаточных напряжений заданной величины, а сам метод калибровки оказывается удобным и довольно широко распространенным для различных способов поверхностно упрочняющей обработки. Источником остаточных напряжений в данном случае является пограничный слой пластических деформаций, наводимый рассматриваемым технологическим процессом. Для постановки задачи задается структура поля тензора пластических деформаций. Форма и напряженно-деформированное состояние упругой пластины с пограничным слоем пластических деформаций были рассчитаны численно, в результате чего были выявлены

\section{Научная статья}

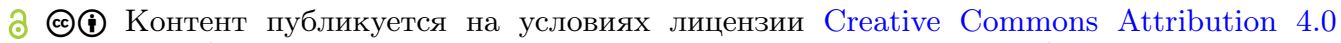
International (https://creativecommons.org/licenses/by/4.0/deed.ru)

\section{Образец для цитирования}

Келлер И. Э., Т рофимов В. Н., Владыкин А. В., Плюснин В. В., Петухов Д. С., В инд ок у ров И. В. К вопросу о реконструкции остаточных напряжений и деформаций пластины после дробеструйной обработки // Вестн. Сам. гос. техн. ун-та. Сер. Физ.мат. науки, 2018. Т. 22, № 1. С. 40-64. doi: 10.14498/vsgtu1602.

\section{Сведения об авторах}

Илья Эрнстович Келлер (10) http://orcid.org/0000-0001-9914-8870

доктор физико-математических наук, доцент; профессор, каф. динамики и прочности машин ${ }^{1}$; научный сотрудник, лаб. нелинейной механики деформируемого твердого тела ${ }^{2}$; e-mail: kie@icmm.ru

Виктор Николаевич Тробимов (1) http://orcid.org/0000-0001-6655-0257 доктор технических наук, доцент; профессор; каф. динамики и прочности машин; e-mail: tvn_perm@mail.ru

Алексей Валеръевич Владыкин; кандидат технических наук; заместитель начальника; центр прогрессивных технологий; e-mail: vladykin-av@pmz.ru 
качественные особенности данных полей, ослаблены граничные условия задачи и сформулированы гипотезы о структуре решения соответствующей пространственной задачи теории упругости, которое далее было найдено аналитически. Показано, что в рамках приближения плоского напряженного состояния в поперечных направлениях результат точно соответствует формуле Давиденкова-Биргера, связывающей зависимость тангенциальной компоненты остаточных напряжений от координаты по толщине пластины с функцией прогибов. Получена явная формула для зависимости остаточной (пластической) деформации от координаты по толщине. Проанализированы источники погрешностей полученных выражений и способы их коррекции. Проведен эксперимент по односторонней дробеструйной обработке калибровочной пластины, изготовленной из закаленной стали $65 \Gamma$, для которой выполнено травление обработанной поверхности с измерением изменения стрелы прогиба (метод Н. Н. Давиденкова). С помощью полученных экспериментальных данных были численно реконструированы профили остаточных напряжений и деформаций с разумной точностью. Результат применим к широкому классу задач для упругих тел с упрочняющими покрытиями, а также имеет определенную методическую ценность для усовершенствования основ экспериментального исследования таких задач, позволяет формулировать и подтверждать экспериментом гипотезы о структуре решения, изучать связь рассматриваемых полей в предельных случаях, верифицировать применение различных способов учета остаточных напряжений и деформаций в численных расчетах. Найденное решение может быть использовано для верификации полей напряжений и перемещений при выборе различных вариантов предварительно напряженных или деформированных поверхностных оболочечных конечных элементов в рамках пакетов прикладных программ для расчета усталостной долговечности деталей машин с поверхностными упрочняющими покрытиями и также представляется опорным для исследования поверхностно упрочненных тел с криволинейной свободной границей, к которым сводится большинство практически важных задач.

Ключевые слова: остаточные напряжения, пограничный слой, дробеструйная обработка, поверхностная обработка, эксперимент, расчет, точное решение, реконструкция.

Получение: 16 января 2018 г. / Исправление: 27 февраля 2018 г. / Принятие: 12 марта 2018 г. / Публикация онлайн: 29 марта 2018 г.

Виктор Валентинович Плюснин; начальник; бюро физико-механических исследований; e-mail: dolgih-nv@pmz.ru

Дмитрий Сергеевич Петухов (D) http://orcid.org/0000-0002-6996-3580

ассистент, каф. динамики и прочности машин ${ }^{1}$; аспирант, лаб. нелинейной механики деформируемого твердого тела ${ }^{2}$; e-mail: petuhovds@mail.ru

Илья Владимирович Виндокуров (D) http://orcid.org/0000-0002-1885-0404

студент; каф. динамики и прочности машин; e-mail: dpmm17@mail.ru 
Введение. Современные регламенты проектирования авиационного двигателя содержат требования прямого учета в расчетах на прочность поверхностного слоя тангенциальных сжимающих остаточных напряжений. Данный слой обычно наводится процессом холодной обработки детали путем бомбардировки участка ее поверхности мелкими стальными шариками. Удар каждой дробинки оставляет на поверхности микроскопическую ямку и в целом покрытие такими ямками приводит к пластическому растяжению материала в касательной плоскости к обрабатываемой поверхности в тонком приповерхностном слое (при обработке пластины в умеренном количестве это ведет к ее изгибу с выпуклостью со стороны обработанной поверхности [1]). Упругость материала стремится восстановить начальную форму детали вблизи ее поверхности, что приводит к образованию в поверхностном слое поля тангенциальных сжимающих остаточных напряжений и необходимого для равновесия поля остаточных напряжений в детали вне этого слоя. Поскольку усталостное и коррозионное разрушение, водородное охрупчивание, разрушение вследствие износа, выкрашивания, образования задиров и кавитационной эрозии деталей машин начинается с поверхности, такая ее обработка способствует увеличению их ресурса. Рассматриваемые технологические процессы поверхностного упрочнения разнообразны, чаще всего используются пневмои гидродробеструйная обработка [2], дробеструйная обработка и обкатка в сочетании ультразвуком [3,4], технологии с применением кавитации [5], обкатка роликом, алмазное выглаживание. Указанные технологии эффективно применяются для литых или спеченных из порошка высокопрочных сталей, алюминиевых и титановых сплавов [6].

В литературе представлены теоретические исследования [7-10], предсказывающие профиль остаточных напряжений в зависимости от параметров процесса на основе рассмотрения единичного удара шара о плоскость в упруго-пластической постановке. Более современные работы [3, 5, 11-18] посвящены прямому моделированию технологического процесса, посредством которого наводятся остаточные напряжения, и который сводится к довольно сложной динамической упругопластической задаче. Данные постановки необходимы для исследования закономерностей формирования остаточных напряжений различными технологическими операциями, выбора рациональных режимов обработки.

Отдельно располагаются задачи расчета ресурса и долговечности поверхностно упрочненных деталей машин в условиях эксплуатации. Для постановки подобных задач вместо расчета предшествующего процесса поверхностного упрочнения разумно задавать пограничный слой остаточных деформаций, который необходимо уметь реконструировать с помощью подходящего метода. В работах [19-21] реконструкция поля остаточных напряжений в объекте предусматривает вспомогательный эксперимент на образце-свидетеле. Для цилиндрических деталей определение остаточных напряжений в поверхностном слое выполняется методом колец и полосок [19,22, 23], основанным на аналитическом решении задачи об остаточных напряжениях в цилиндрическом теле [19]. В качестве образца-свидетеля используется сплошной или полый цилиндр одинакового с деталью диаметра. Данный подход позволяет решать задачи об усталостной долговечности валов в галтельными переходами и выточками при циклическом изгибе, кручении, растяжении-сжатии [20], 
а также с учетом релаксации остаточных напряжений в условиях ползучести $[21,24]$. В работах $[25,26]$ используется термоупругая аналогия для учета остаточных напряжений при конечно-элементном расчете прочности цилиндрических деталей.

Сведение краевой задачи реконструкции напряженно-деформированного состояния в области концентратора напряжений в форме полукругового надреза после опережающего поверхностно пластического упрочнения к фиктивной задаче термоупругости с неоднородным полем температуры и неоднородным анизотропным полем тензора коэффициентов температурного расширения рассмотрено в работах [27] для сплошных и [28] полых цилиндрических образцов.

На производстве необходимая длительность дробеструйной обработки детали определяется с помощью пластинки, прогиб которой калибруют в соответствии с уровнем остаточных напряжений, определяемым экспериментально. Данный метод калибровки является удобным и довольно широко распространенным для различных способов поверхностно упрочняющей обработки $[1,29]$; одна из его разновидностей запатентована в США и называется тестом Ольмена (Almen Strip Test) [30].

Целью настоящей работы является постановка задачи определения напряженно-деформированного состояния пластинки с заданной структурой пограничного слоя пластических деформаций, построение точного или приближенного аналитического решения данной задачи и его экспериментальное подтверждение. Данный результат необходим для решения обратной задачи реконструкции приповерхностного распределения остаточных деформаций и напряжений по определяемому в лаборатории прогибу пластины в зависимости от толщины стравливаемого слоя материала с обработанной поверхности. Поле остаточных (пластических) деформаций служит источником остаточных напряжений и необходимо для учета последних при расчетах напряженно-деформированного состояния деталей машин. Рассматриваемая задача для пластины вследствие своей простоты имеет определенную методическую ценность, поскольку позволяет сформулировать и подтвердить экспериментом гипотезы о структуре решения, изучить связь рассматриваемых полей в асимптотических и предельных случаях, верифицировать применение различных способов учета остаточных напряжений в численных расчетах. Искомое решение также представляется опорным для исследования поверхностно упрочненных тел с криволинейной свободной границей, к которым сводится большинство практически важных задач. Современные методы реконструкции остаточных напряжений опираются на решения [19], полученные методами сопротивления материалов, в результате чего ускользает понимание структуры распределения остаточных напряжений, пределов применимости формул и возможности их уточнения. Упругая задача для плиты и полосы, вообще говоря, представляет собой большую аналитическую сложность [31-34], следовательно, рассматриваемая здесь проблема требует к себе серьезного отношения.

\section{1. Эксперимент по односторонней дробеструйной обработке пла-} стины. На АО «ОДК-Пермские моторы» было организовано экспериментальное исследование напряженно-деформированного состояния пластины после односторонней дробеструйной обработки. Калибровочная пластина из 
конструкционной рессорно-пружинной стали 65Г, закаленной до твердости HRC 51, размерами $100 \times 19 \times 1.3$ мм, консольно закрепленная на одном конце и свободно опертая на другом, была обработана с одной стороны микрошариками из распыленного порошка быстрорежущих сталей фракции 0.16-0.20 на пневмодробеструйной установке типа $\oslash 6397-4330$ в течение 45 секунд без вращения. Обработанная пластина приобрела изогнутую форму с выпуклостью навстречу воздействию со стрелой прогиба 0.32 мм на базе 60 мм длины, измеренной согласно принятой методике. Из средней части этой пластины продольной электроэрозионной вырезкой были изготовлены три образца-свидетеля размерами $54 \times 5.9 \times 1.29$ мм. Для каждого из них на установке АПООН [35] методом Н. Н. Давиденкова было определено распределение остаточных напряжений по глубине. Для этого с базовой длины 18 мм средней части упрочненной грани образца автоматически стравливался металл, измерялось изменение стрелы прогиба и реконструировалось распределение тангенциальной компоненты напряжений по глубине. При снятии слоя глубиной 200 мкм прогиб практически полностью прекращал изменяться. В пределах данного слоя располагались пластические деформации, являющиеся источником остаточных напряжений и вызванного ими прогиба. На рис. 1 приведены экспериментальные зависимости изменения стрелы прогиба от толщины снятого слоя, соответствующие каждому из образцов.

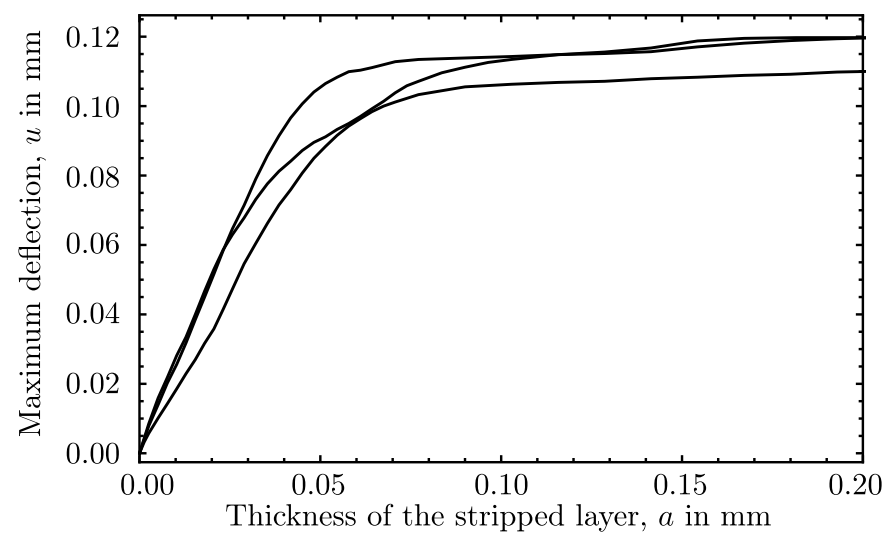

Рис. 1. Зависимость стрелы прогиба от толщины снятого слоя для трех образцов (экспериментальные данные) [Figure 1. The maximum deflection of a plate after removal of the layer for three samples (experimental data)]

2. Уравнения и гипотезы. При построении уравнений принимается гипотеза о том, что упругие свойства в пограничном слое, охваченном пластическими деформациями, после дробеструйной обработки остаются изотропными и характеризуются теми же упругими модулями, какие были до обработки. Модель в сильной (дифференциальной) постановке включает в себя уравнения равновесия упругого тела с пластическими деформациями:

$$
\begin{gathered}
\boldsymbol{\nabla} \cdot \boldsymbol{\sigma}=\mathbf{0}, \quad \boldsymbol{\sigma}=\lambda \operatorname{tr}\left(\boldsymbol{\varepsilon}^{e}\right) \boldsymbol{I}+2 \mu \boldsymbol{\varepsilon}^{e}, \\
\boldsymbol{\varepsilon}^{e}=\boldsymbol{\varepsilon}-\boldsymbol{\varepsilon}^{p}, \quad \boldsymbol{\varepsilon}=\frac{1}{2}(\boldsymbol{\nabla} \boldsymbol{u}+\boldsymbol{u} \boldsymbol{\nabla}), \quad \operatorname{tr}\left(\boldsymbol{\varepsilon}^{p}\right)=0 ;
\end{gathered}
$$

условия на боковых сторонах пластины:

$$
\boldsymbol{n} \cdot \boldsymbol{\sigma}=\mathbf{0} \quad(z=0, z=h)
$$


условия на ее концах:

$$
\boldsymbol{n} \cdot \boldsymbol{\sigma}=\mathbf{0} \quad(x= \pm l)
$$

и условия закрепления:

$$
u_{x}=u_{y}=0 \quad(x=y=0), \quad u_{z}=0 \quad(x=y=z=0) .
$$

Здесь $\boldsymbol{\sigma}$ - тензор напряжений; $\boldsymbol{\varepsilon}, \boldsymbol{\varepsilon}^{e}, \boldsymbol{\varepsilon}^{p}-$ тензоры полных, упругих и пластических деформаций; $\boldsymbol{u}$ - вектор перемещений; $\boldsymbol{n}$ - внешняя нормаль к границе; $x, y, z$ - декартовы координаты, последняя из которых перпендикулярна плоскости пластинки (рис. 2).

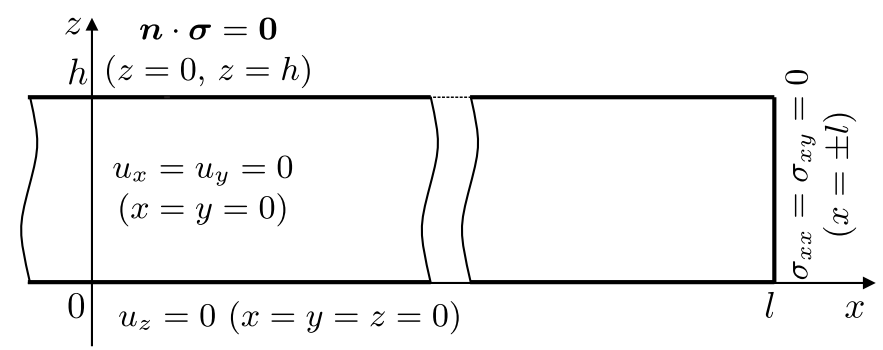

Рис. 2. Постановка задачи для пластины конечных размеров

[Figure 2. The statement of the problem for a finite plate]

Уравнения (1)-(4) могут быть записаны в форме

$$
\begin{gathered}
\mu \boldsymbol{\nabla} \cdot \boldsymbol{\nabla} \boldsymbol{u}+(\lambda+\mu) \boldsymbol{\nabla} \boldsymbol{\nabla} \cdot \boldsymbol{u}=2 \mu \boldsymbol{\nabla} \cdot \boldsymbol{\varepsilon}^{p}, \\
\lambda \boldsymbol{n} \boldsymbol{\nabla} \cdot \boldsymbol{u}+\mu \boldsymbol{n} \cdot(\boldsymbol{\nabla} \boldsymbol{u}+\boldsymbol{u} \boldsymbol{\nabla})=2 \mu \boldsymbol{n} \cdot \boldsymbol{\varepsilon}^{p}, \quad z=0, h ; x= \pm l,
\end{gathered}
$$

из которой видно, что поле пластических деформаций трансформируется в массовые и поверхностные силы и является источником остаточных полей упругих перемещений, деформаций и напряжений.

Принимается гипотеза о следующей структуре тензора пластических деформаций:

$$
\varepsilon_{x x}^{p}=\varepsilon_{y y}^{p}=-\frac{1}{2} \varepsilon_{z z}^{p}=f(z), \quad \varepsilon_{x y}^{p}=\varepsilon_{x z}^{p}=\varepsilon_{y z}^{p}=0,
$$

где $f(z)$ - функция, задающая пограничный слой пластических деформаций, формируемый односторонней дробеструйной обработкой. На рис. 3 для примера приведена функция

$$
f(z)=a(1+\operatorname{th}(k(z-b))),
$$

где $a=0.5, b=0.9, k=20$, изменяющаяся в пределах от 0 до $2 a$ на отрезке $[b-2 / k, b+2 / k]$ и качественно отражающая структуру пограничного слоя Следует заметить, что тензор $\varepsilon^{p}$, источник остаточных напряжений, можно трактовать как тензор деформаций пластины с неоднородным анизотропным полем тензора коэффициентов линейного температурного расширения под действием однородного поля температуры:

$$
f(z)=k(z) \Delta T
$$




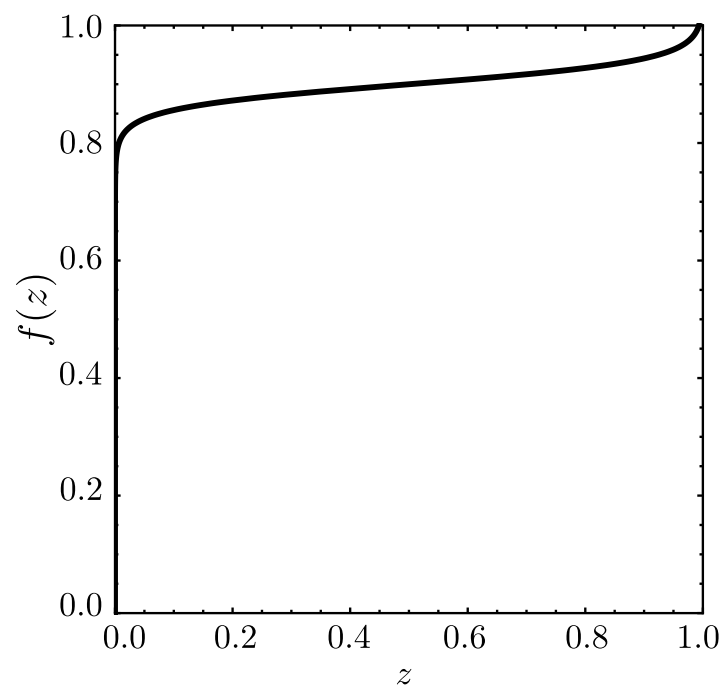

Рис. 3. Профиль пограничного слоя пластических деформаций

[Figure 3. The profile $f(z)$ of the boundary layer (see Eq. (7), where $a=0.5, b=0.9, k=20$ ) of the plastic strain as a function of the plate thickness $z$ ]

Поле тензора пластических деформаций, имеющего структуру (6), является несовместным, то есть порождает остаточные напряжения, если выражение

$$
\operatorname{Ink} \varepsilon^{p}=\nabla \times \varepsilon^{p} \times \nabla=f^{\prime \prime}(z)\left(\boldsymbol{a}_{x} \boldsymbol{a}_{x}+\boldsymbol{a}_{y} \boldsymbol{a}_{y}\right),
$$

где $\boldsymbol{a}_{x}, \boldsymbol{a}_{y}$-ортонормированный базис в плоскости пластины, тождественно не равно нулю на отрезке $z \in[0, h]$. Это выполняется, если функция $f(z)$ на этом отрезке не является линейной. Функция (7), как и более простая двухпараметрическая зависимость

$$
f(z)=\left\{\begin{array}{cl}
0, & z \in\left[0, z_{*}\right] ; \\
k\left(z-z_{*}\right), & z \in\left[z_{*}, h\right],
\end{array}\right.
$$

для которой $\operatorname{Ink} \varepsilon^{p} \neq \mathbf{0}$ в одной точке $z=z_{*}$, гарантированно удовлетворяют такому условию.

3. Численный расчет и качественный анализ решения. С целью качественного анализа напряженно-деформированного состояния пластины с пограничным слоем пластических деформаций $(6),(7)$ задача $(1),(2),(4)$, переформулированная в слабой постановке для плоско-деформированного состояния, была численно решена в пакете ANSYS Mechanical 14. В расчетах источник остаточных напряжений задавался в термоупругой постановке с однородным полем температуры $\Delta T$ и неоднородным анизотропным полем тензора коэффициентов линейного температурного расширения (8). Размеры пластины $100 \times 1.3$ мм, модуль Юнга $E=215$ ГПа и коэффициент Пуассона $\nu=0.34$ соответствовали размерам и материалу калибровочной пластины в эксперименте.

Деформация и распределение напряжений $\sigma_{x x}$ в пластине с полем пластических деформаций $\varepsilon^{p}$ приведены на рис. 4,5 . Пластина оказывается выпуклой со стороны, на которой находится пограничный слой пластических 
деформаций, при этом компонента перемещений $u_{x}$ в сечении, взятом на расстоянии одной четвертой длины пластины для исключения краевых эффектов (рис. 6), оказывается линейно зависящей от координаты $z$, то есть кинематика пластины соответствует гипотезе плоских сечений. Значение стрелы прогиба на базе 60 мм длины пластины оказалось равным 0.21 мм.

Профиль компоненты $\sigma_{x x}$ остаточных напряжений в сечении, взятом на расстоянии одной четвертой длины пластины (для исключения краевых эффектов), приведен на рис. 7. Здесь обращают на себя внимание следующие особенности. Заданный источник $\varepsilon^{p}$ создает поле сжимающего тангенциального напряжения вблизи обеих наружных поверхностей пластинки, а также поле растягивающего тангенциального напряжения во внутренней области пластинки так, что интегральные значения силы и момента этого поля по толщине пластинки оказываются практически нулевыми:

$$
F=\int_{0}^{h} \sigma_{x x}(z) d z \approx 0, \quad M=\int_{0}^{h} z \sigma_{x x}(z) d z \approx 0
$$

Зависимости

$$
F(z)=\int_{0}^{z} \sigma_{x x}(\zeta) d \zeta, \quad M(z)=\int_{0}^{z} \zeta \sigma_{x x}(\zeta) d \zeta
$$

приведены на рис. 8, 9 .

Профиль исследуемой компоненты остаточных напряжений оказывается линейным вне пограничного слоя и в определенном масштабе с инверсией повторяет заданный профиль пластических деформаций в пределах пограничного слоя. По этой причине остаточные напряжения меняют знак в точке, располагающейся в пределах пограничного слоя пластических деформаций.

4. Построение аналитического решения. Численный анализ задачи (1), (2), (4), (6), (7), выполненный в предыдущем разделе, позволяет рассматривать упрощенную формулировку задачи для определения напряженно-деформированного состояния поверхностно упрочненной калибровочной пластины, игнорирующую небольшие краевые эффекты.

Рассмотрим неограниченную пластину (рис. 10), для которой вместо граничных условий (3) принимаются условия

$$
\int_{0}^{h} \sigma_{r r}(z) d z=0, \quad \int_{0}^{h} z \sigma_{r r}(z) d z=0, \quad r>0,
$$

где $r, \varphi, z$ - цилиндрические координаты, последняя из которых перпендикулярна плоскости пластинки. Условия закрепления (4) для нее формулируются в виде

$$
\left.u_{r}\right|_{r=0}=0,\left.\quad u_{z}\right|_{\substack{r=0 \\ z=0}}=0,\left.\quad \omega\right|_{r=0}=0
$$

где $\omega$ - малый поворот в плоскости пластины, и компоненты тензора пластических деформаций (6) переписываются следующим образом:

$$
\varepsilon_{r r}^{p}=\varepsilon_{\varphi \varphi}^{p}=-\frac{1}{2} \varepsilon_{z z}^{p}=f(z), \quad \varepsilon_{r \varphi}^{p}=\varepsilon_{r z}^{p}=\varepsilon_{\varphi z}^{p}=0 .
$$




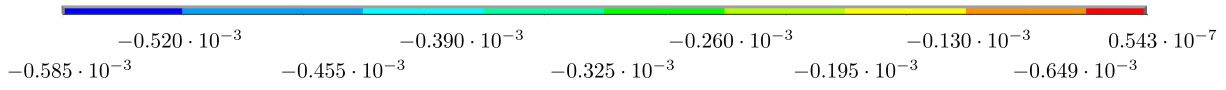

Рис. 4. Рассчитанное искривление пластины, вызванное пограничным слоем пластических деформаций [Figure 4. The calculated plate bending caused by a boundary layer of the plastic strains]

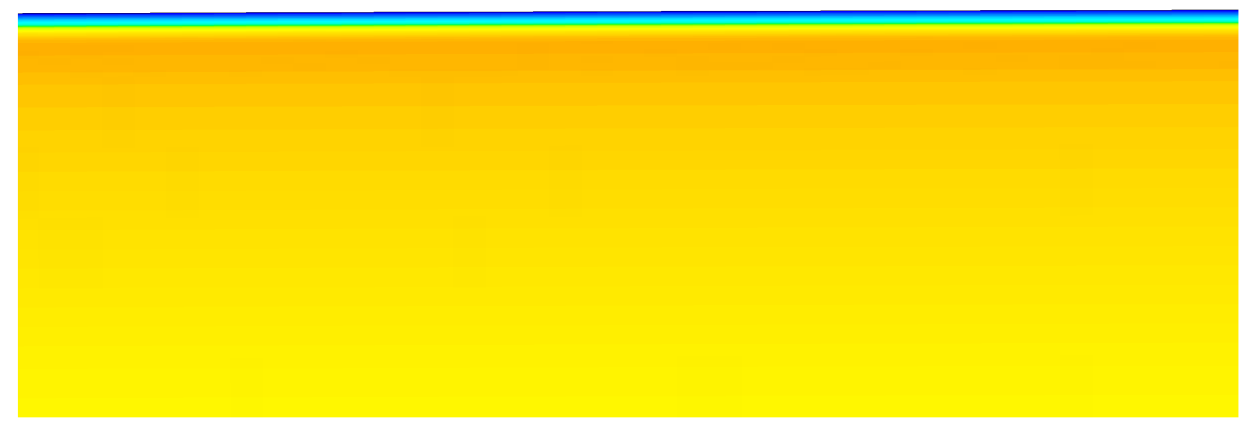

\begin{tabular}{rlllllllll}
\hline-724.82 & -627.15 & -529.47 & -431.79 & -334.11 & -236.43 & -138.75 & -41.08 & 56.59 & 168.23
\end{tabular}

Рис. 5. Рассчитанное распределение остаточных напряжений $\sigma_{x x}$ (МПа), вызванное пограничным слоем пластических деформаций [Figure 5. The calculated residual stresses distribution, $\sigma_{x x}$ in $\mathrm{MPa}$, caused by a boundary layer of the plastic strains]

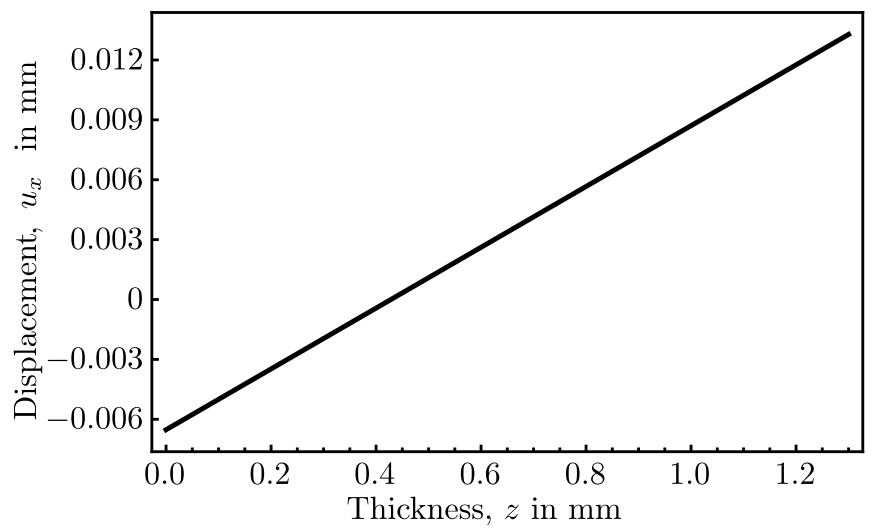

Рис. 6. Рассчитанный профиль тангенциальной компоненты перемещений $u_{x}$ по толщине пластины [Figure 6. The calculated tangential component of displacements, $u_{x}$ in mm, as a function of the plate thickness $z$ ] 


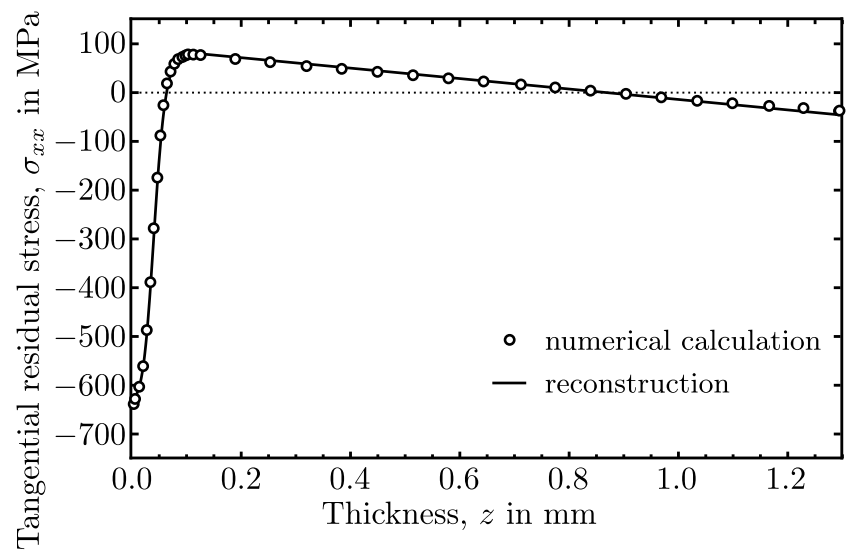

Рис. 7. Рассчитанный профиль тангенциальной компоненты остаточных напряжений по толщине пластины в сопоставлении с экспериментальными данными

[Figure 7. Calculated and reconstructed tangential residual stress fields across the plate thickness]

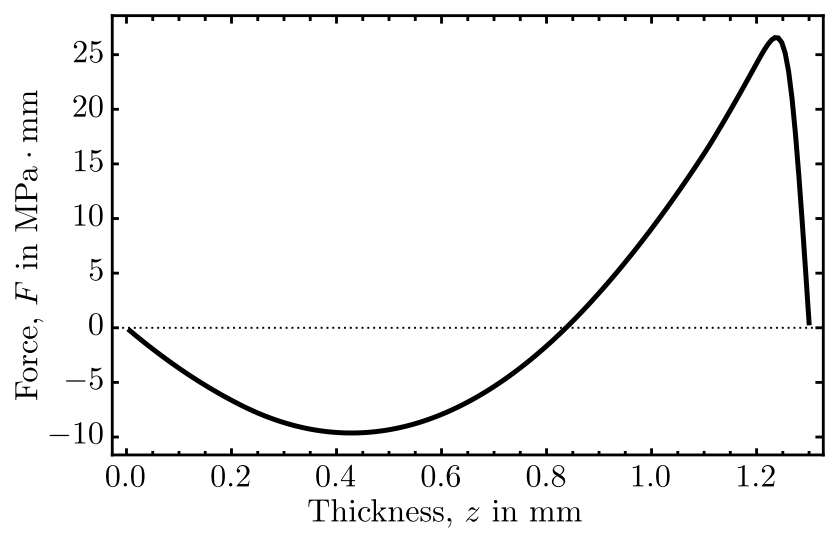

Рис. 8. Зависимость силы $F$ в поперечном сечении $x=l / 2$ от координаты $z$

[Figure 8. The force distribution in a cross-section through the plate thickness for $x=l / 2$ ]

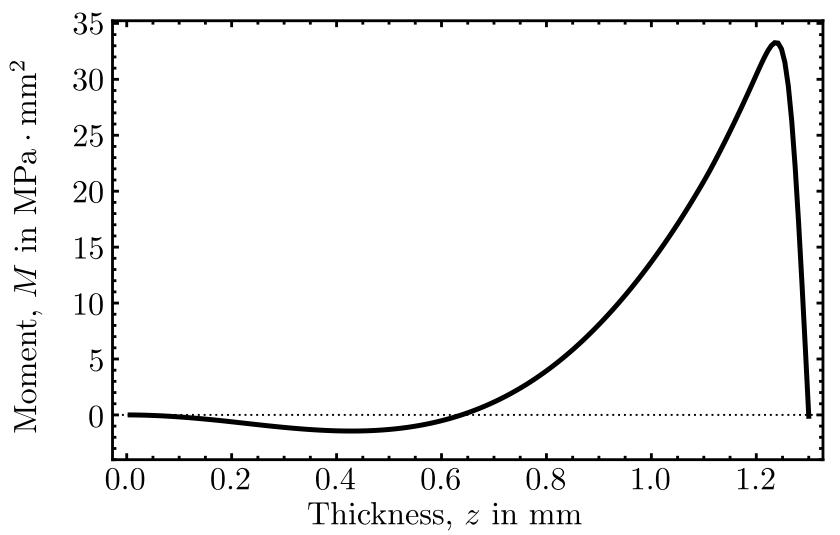

Рис. 9. Зависимость момента $M$ в поперечном сечении $x=l / 2$ от координаты $z$

[Figure 9. The moment variation in a cross-section through the plate thickness for $x=l / 2$ ] 


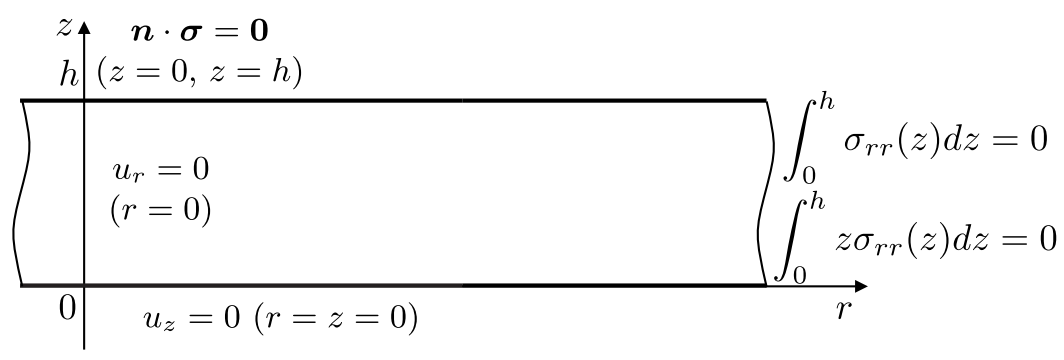

Рис. 10. Постановка задачи для неограниченной пластины [Figure 10. The statement of the problem for an infinite plate]

Характер механической обработки калибровочной пластины позволяет предположить, что пластические деформации (7), (12) порождают поле полных деформаций, которое является однородным и изотропным в плоскости пластины (тензор полных деформаций осесимметричный [36] с осью симметрии, нормальной к плоскости пластины, и зависит только от координаты $z$ ):

$$
\varepsilon_{r r}=\varepsilon_{\varphi \varphi}=\varepsilon_{1}(z), \quad \varepsilon_{z z}=\varepsilon_{2}(z), \quad \varepsilon_{r \varphi}=\varepsilon_{r z}=\varepsilon_{\varphi z}=0 .
$$

В таком случае из уравнений совместности деформаций Ink $\varepsilon=0$ (определение оператора несовместности см. в (9)) следует

$$
\varepsilon_{1}(z)=C z+D,
$$

то есть выполняется гипотеза плоских сечений. Далее из уравнений равновесия, упругих соотношений системы (1) и граничного условия (2) с учетом (6), (13), (14), перейдя к техническим упругим константам, можно выписать следующие выражения для компонент напряжений:

$$
\begin{gathered}
\sigma_{r r}(z)=\sigma_{\varphi \varphi}(z)=-\frac{E}{1-\nu}(f(z)-C z-D), \\
\sigma_{r \varphi}=\sigma_{\varphi z}=\sigma_{r z}=\sigma_{z z} \equiv 0 .
\end{gathered}
$$

Произвольные константы $C$ и $D$ определяются из условий (10):

$$
\begin{gathered}
C h=12 N-6 K, \quad D=4 K-6 N ; \\
K=\frac{1}{h} \int_{0}^{h} f(z) d z, \quad N=\frac{1}{h^{2}} \int_{0}^{h} z f(z) d z .
\end{gathered}
$$

Одновременно с напряжениями конкретизируется выражение

$$
\varepsilon_{2}(z)=-2 C \frac{\lambda}{\lambda+2 \mu} z-2 D \frac{\lambda}{\lambda+2 \mu}-4 \frac{\mu}{\lambda+2 \mu} f(z) .
$$

Следует отметить, что условия (10) можно получить из требования минимальности энергии остаточных напряжений (на единицу площади пластины)

$$
U=\frac{E}{1-\nu} \int_{0}^{h}(C z+D-f(z))^{2} \rightarrow \min
$$


по произвольным константам $C, D$ (соответствующий вариационный принцип приводится в [37, раздел 4.4]). Таким образом, качественные особенности напряженно-деформированного состояния, выявленные с помощью численного расчета, по сути, вытекают из гипотез (13), определяющих решение.

Далее по найденным на предыдущем этапе выражениям для $\varepsilon_{1}(z)$ и $\varepsilon_{2}(z)$ из кинематических соотношений системы (1) и условий (11) определяются компоненты перемещений. Сначала из уравнения

$$
\varepsilon_{z}=\frac{\partial u_{z}}{\partial z}=\varepsilon_{2}(z)
$$

находится

$$
u_{z}=\int_{0}^{z} \varepsilon_{2}(z) d z+g(r, \varphi),
$$

где $g(r, \varphi)$ - произвольная функция. Затем из условия

$$
\left.\omega\right|_{r=0}=\left.\frac{1}{2}\left(\frac{\partial u_{\varphi}}{\partial r}=\frac{1}{r} \frac{\partial u_{r}}{\partial \varphi}+\frac{u_{\varphi}}{r}\right)\right|_{r=0}=0
$$

с учетом (14) находится $u_{\varphi} \equiv 0$. С учетом этого из уравнения

$$
\varepsilon_{\varphi \varphi}=\frac{1}{r} \frac{\partial u_{\varphi}}{\partial \varphi}+\frac{u_{r}}{r}=\varepsilon_{1}(z)
$$

следует $u_{r}=(C z+D) r$.

Далее из уравнения

$$
\varepsilon_{\varphi z}=\frac{1}{2}\left(\frac{\partial u_{\varphi}}{\partial z}+\frac{1}{r} \frac{\partial u_{z}}{\partial \varphi}\right)=0
$$

вытекает, что $g(r, \varphi) \equiv g(r)$. Наконец, из уравнения

$$
\varepsilon_{z r}=\frac{1}{2}\left(\frac{\partial u_{z}}{\partial r}+\frac{\partial u_{r}}{\partial z}\right)=0
$$

с учетом (14), последнего из условий (11) и полученной выше конкретизации функции $g$ вытекает $g(r)=-\frac{C}{2} r^{2}$, и, окончательно, перейдя к техническим упругим константам, компоненты перемещений можно записать в виде

$$
\begin{gathered}
u_{r}=C r z+D r, \quad u_{\varphi} \equiv 0 \\
u_{z}=-\frac{C}{2} r^{2}-\frac{C \nu}{1-\nu} z^{2}-\frac{2 D \nu}{1-\nu} z-\frac{2(1-2 \nu)}{1-\nu} \int_{0}^{z} f(z) d z .
\end{gathered}
$$

Из последнего выражения находится прогиб пластины:

$$
\left.u_{z}\right|_{\substack{r=l \\ z=0}}=-\frac{C}{2} l^{2}=-\frac{3 l^{2}}{h}(2 N-K) .
$$

Из (15) следует, что профиль пограничного слоя остаточных напряжений в пластине состоит из слагаемого, конгруэнтного профилю пограничного слоя 
пластических деформаций, и линейного слагаемого с произвольными постоянными, определяемыми из интегральных условий равенства нулю усилия и момента в пластине. Структура решения подтверждает вывод о том, что остаточные напряжения меняют знак в точке, располагающейся в пределах пограничного слоя пластических деформаций.

В практически важных случаях отношение $\delta=\hat{h} / h$, где $\hat{h}$-характерная толщина упрочненного пограничного слоя, является малым параметром. Из соотношений (15), (16) следует, что постоянные $C, D$, определяющие линейную часть выражения остаточных напряжений, суть малые величины порядка $\delta$. При неограниченном увеличении толщины пластины при любом фиксированном значении $\hat{h}$ постоянные $C, D$ стремятся к нулю вместе с $\delta$, в силу чего выражение (15) трансформируется в формулу

$$
\sigma_{r r}=\sigma_{\varphi \varphi}=-\frac{E}{1-\nu} f(z)
$$

полученную Ж. Заркой [38] (вывод которой можно найти в более доступной работе [13]).

Выражения (12), (14), (15), (17) позволяют установить, что напряжения в постановке задачи (5)-(8)

$$
\sigma_{r r}^{\prime}=\sigma_{\varphi \varphi}^{\prime}=\frac{E}{1-\nu}(C z+D)-\frac{E}{1-\nu} \frac{2 \nu}{1+\nu} f(z)
$$

отличаются от остаточных напряжений (15):

$$
\sigma_{r r}=\sigma_{r r}^{\prime}-\frac{E}{1+\nu} f(z), \quad \sigma_{\varphi \varphi}=\sigma_{\varphi \varphi}^{\prime}-\frac{E}{1+\nu} f(z)
$$

Несложно показать, что в выражениях для $\sigma_{r r}^{\prime}, \sigma_{\varphi \varphi}^{\prime}$ для превращения их в остаточные напряжения $\sigma_{r r}, \sigma_{\varphi \varphi}$ следует умножить слагаемое $f(z)$ на множитель $\frac{1+\nu}{2 \nu}$. Эту операцию необходимо выполнять при численном расчете остаточных напряжений при использовании постановки (5)-(8). Использование представления пластических деформаций остаточными или термоупругими деформациями (13) в стандартных типах конечных элементов пакета ANSYS не требует корректировки напряжений.

5. Обоснование формулы Давиденкова-Биргера. Для реконструкции остаточных напряжений в призматическом теле методом Давиденкова обычно используют формулу Давиденкова-Биргера [19, с. 65-67]:

$$
\sigma_{x x}(z)=-\frac{E}{3 l^{2}}\left(z^{2} u^{\prime}(z)+4 z(u(z)-u(h))+2 \int_{h}^{z}(u(\zeta)-u(h)) d \zeta\right)
$$

выведенную в приближении теории изгиба балок. В выражении (19) u(z) зависимость стрелы прогиба балки от координаты $z$ верхней грани балки после стравливания слоя толщиной $h-z$.

Для реконструкции остаточных напряжений в круглой или квадратной пластине может быть использовано решение (15), (16), (18), однако для призматического тела корректнее использовать более подходящие постановки за- 
дачи. Для условия плоского деформированного состояния $\boldsymbol{u}=\boldsymbol{u}(x, z)$ рассматривается следующая структура тензора пластических деформаций:

$$
\varepsilon_{x x}^{p}=-\varepsilon_{z z}^{p}=f(z), \quad \varepsilon_{y y}^{p}=0, \quad \varepsilon_{x y}^{p}=\varepsilon_{x z}^{p}=\varepsilon_{y z}^{p}=0 .
$$

Поскольку такой тензор является ортотропным [36], задачу удобнее рассматривать в декартовой ортогональной системе координат. В граничных условиях (10) под $\sigma_{r r}$ следует понимать $\sigma_{x x}$. Ненулевые компоненты остаточных напряжений принимают вид

$$
\sigma_{x x}=-\frac{E}{1-\nu^{2}}(f(z)-C z-D), \quad \sigma_{y y}=\frac{E \nu}{1-\nu^{2}}(C z+D),
$$

а выражения (16), (18) остаются без изменений.

Для плоского напряженного состояния принимается следующая структура тензора пластических деформаций:

$$
\varepsilon_{x x}^{p}=f(z), \quad \varepsilon_{y y}^{p}=g(z), \quad \varepsilon_{z z}^{p}=-f(z)-g(z), \quad \varepsilon_{x y}^{p}=\varepsilon_{x z}^{p}=\varepsilon_{y z}^{p}=0,
$$

причем плоское напряженное состояние $\sigma_{y y} \equiv 0$ возможно только в случае

$$
g(z)=-\frac{\lambda}{2(\lambda+\mu)} f(z) .
$$

Ненулевая компонента остаточных напряжений имеет вид

$$
\sigma_{x x}=-E(f(z)-C z-D) ;
$$

выражения (16), (18) также остаются без изменений. Последнее решение соответствует изгибу призматического тела и его корректно сравнить с формулой (19).

Покажем, что формула (19) вытекает из решения (15), (16), (18). Из (18) с учетом (16) получается

$$
f(z)=\frac{1}{3 l^{2}}\left(z^{2} u^{\prime}(z)+4 z u(z)+2 \int_{0}^{z} u(\zeta) d \zeta\right) .
$$

Далее из (21) с учетом (16) и выражений

$$
\begin{aligned}
& \int_{0}^{h} f(\zeta) d \zeta=\frac{1}{3 l^{2}}\left(h^{2} u(h)+2 h \int_{0}^{h} u(\zeta) d \zeta\right) \\
& \int_{0}^{h} f(\zeta) \zeta d \zeta=h^{3} u(h)+\frac{1}{3 l^{2}}\left(h^{2} \int_{0}^{h} u(\zeta) d \zeta\right)
\end{aligned}
$$

вытекающих из (22), после некоторых элементарных манипуляций можно получить (19).

В монографии [39, с. 57-60] определено напряженно-деформированное состояние пластины с остаточными (здесь пластическими) деформациями. Постановка задачи в сравнении с рассматриваемой в настоящей работе содержит дополнительные предположения, вместе с которыми выражения (15), (16) остаются в силе, но выражение для стрелы прогиба принимает вид

$$
\left.u_{z}\right|_{\substack{r \\ z=h / 2}}=-\frac{3}{h}(2 N-K)\left(\frac{1}{2}(1-\nu) l^{2}+(1+\nu) l\right) .
$$


Данное решение также приведено в монографии [19], но для реконструкции остаточных напряжений и обоснования формулы (19) оно не использовалось.

\section{6. Численная реализация процедуры реконструкции остаточных} напряжений и деформаций. В случае стравливания части поверхности образца выражения (19), (22) примут вид

$$
\begin{gathered}
\sigma(a)=\frac{4 E}{3 l\left(2 l_{y}-l\right)}\left((h-a)^{2} u^{\prime}(a)-4(h-a) u(a)-2 \int_{0}^{a} u(\zeta) d \zeta\right), \\
\varepsilon(a)=\frac{4}{3 l\left(2 l_{y}-l\right)}\left(-(h-a)^{2} u^{\prime}(a)+4(h-a)\left(u(a)-u_{0}\right)-\right. \\
\left.-2 \int_{a}^{h}\left(u(\zeta)-u_{0}\right) d \zeta\right),
\end{gathered}
$$

где $\sigma(a)=\sigma_{x x}(z), \varepsilon(a)=f(z), l_{y}$ - длина образца, $l$ - длина фрагмента образца, на котором происходит травление. Здесь для удобства сделана замена $z$ на $h-a$, где $a$-глубина, отсчитываемая относительно упрочненной поверхности, $u(a)$ - изменение стрелы прогиба в процессе стравливания, то есть $u(0)=0, u_{0}=u\left(a_{0}\right)$, где $a_{0}$ - глубина упрочненного слоя, причем $u(a)=u\left(a_{0}\right)$ при $a>a_{0}$, что означает постоянство прогиба при стравливании неупрочненного материала.

По массиву значений изменения стрелы прогиба $u\left(a_{i}\right)=u_{i}, i=1, \ldots, n$, можно реконструировать поля остаточных напряжений и пластических деформаций в образце. Стоит отметить, что значения остаточных напряжений можно реконструировать пошагово, то есть после снятия $k$ слоев можно получить $k$ значений $\sigma_{i}, i=1, \ldots, k$, тогда как реконструкция функции остаточных деформаций возможна только после снятия всех слоев, содержащих пластические деформации, когда станет известен весь массив значений прогиба.

Выражения (23), (24) в дискретном случае имеют вид

$$
\begin{gathered}
\sigma_{i}=\frac{4 E}{3 l\left(2 l_{y}-l\right)}\left(\left(h-a_{i}\right)^{2} u_{i}^{\prime}-4\left(h-a_{i}\right) u_{i}+2 \int_{0}^{a_{i}} u(\zeta) d \zeta\right), \\
\varepsilon_{i}=\frac{4 E}{3 l\left(2 l_{y}-l\right)}\left(-\left(h-a_{i}\right)^{2} u_{i}^{\prime}+4\left(h-a_{i}\right)\left(u_{i}-u_{0}\right)-\right. \\
\left.-2 \int_{a_{i}}^{h}\left(u(\zeta)-u_{0}\right) d \zeta\right) .
\end{gathered}
$$

Значения интегралов в (25), (26) можно получать методом трапеций. Значения производной $u_{i}^{\prime}$ следует находить с высокой точностью, однако даже разностные схемы высокой точности заметно усиливают погрешности экспериментальных данных. Поэтому дискретизованные экспериментальные данные были сначала аппроксимированы гладкой функцией, а затем использованы формулы (23), (24). Для аппроксимации использовалась функция

$$
\tilde{u}(a)=\kappa_{1}\left(\left[1+\operatorname{erf}\left(\kappa_{3}\left(\kappa_{2} a-1\right)\right)\right]+\kappa_{2} a\left[1-\operatorname{erf}\left(\kappa_{3}\left(\kappa_{2} a-\kappa_{4}\right)\right)\right]\right),
$$


где erf - функция ошибок, а коэффициенты $\kappa_{i}$ были найдены методом наименьших квадратов.

На рис. 11 приведена дискретизация одной из кривых прогибов (верхняя кривая на рис. 1) и ее аппроксимация (27).

На рис. 12 и 13 приведены результаты поточечной реконструкции полей остаточных напряжений и пластических деформаций по формулам (25), (26) (для вычисления значений производной использовалась пятиточечная разностная схема), а также результаты реконструкции с использованием аппроксимации (27) по формулам (23), (24).

На рис. 7 приведены результаты численного расчета остаточных напряжений, в качестве источников которых задавалась сглаженная версия реконструированного профиля пластических деформаций (рис. 13).

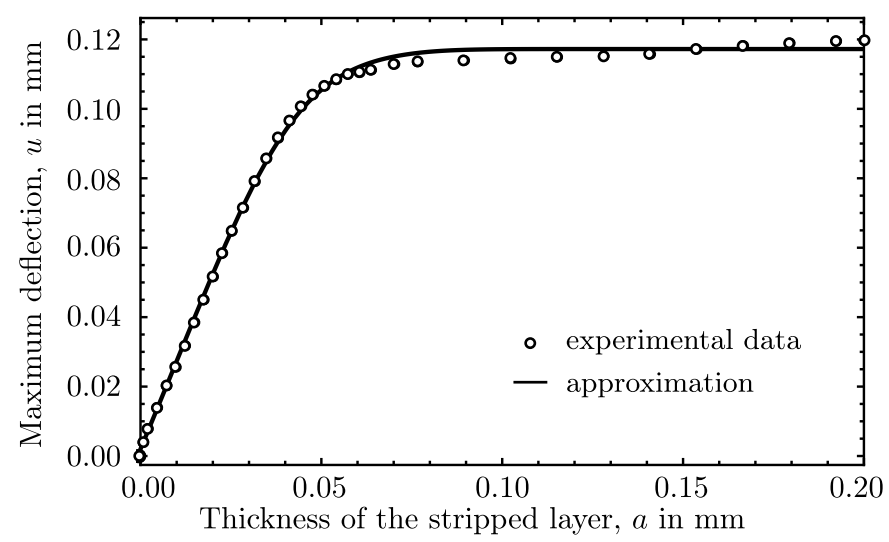

Рис. 11. Зависимость стрелы прогиба от толщины снятого травлением слоя (эксперимент и аппроксимация (27))

[Figure 11. The maximum deflection of a plate after removal of the layer (experimental data and approximation (27) for the top curve in Fig. 1)]

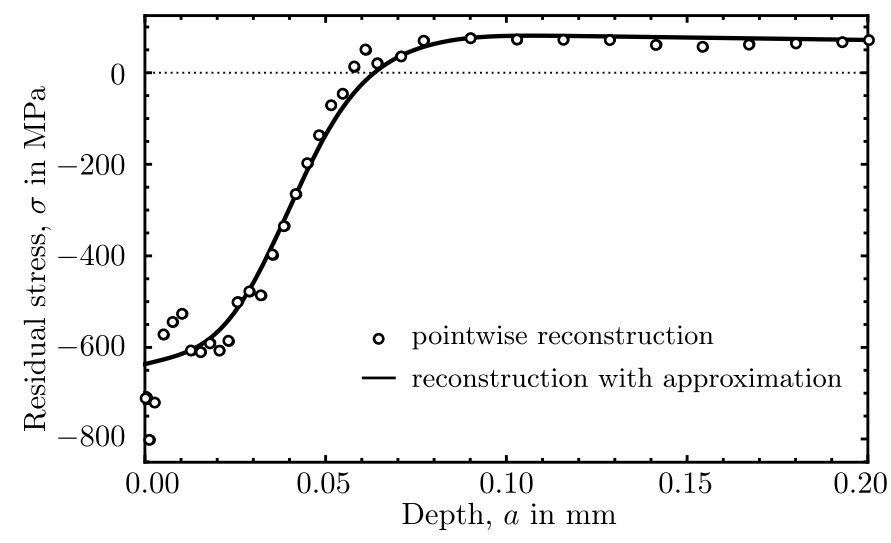

Рис. 12. Остаточные напряжения в пограничном слое, реконструированные по данным эксперимента

[Figure 12. Residual stresses in the boundary layer reconstructed from experimental data] 


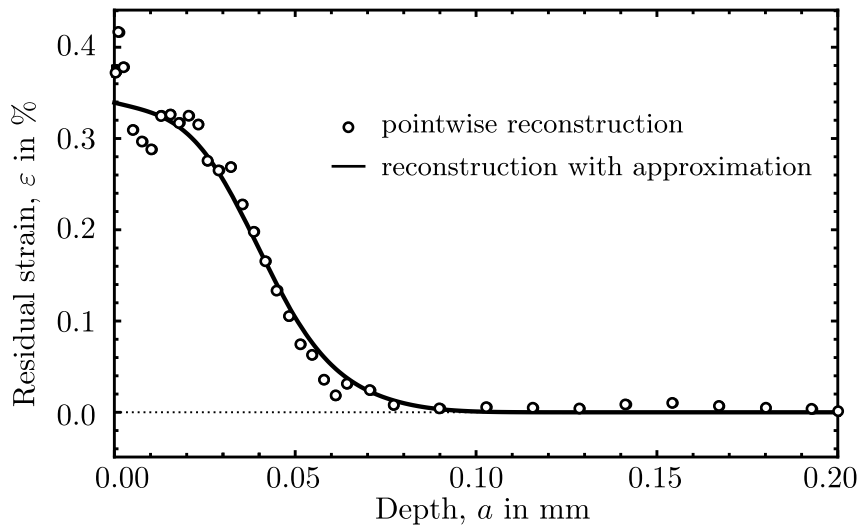

Рис. 13. Остаточные деформации в пограничном слое, реконструированные по данным эксперимента

[Figure 13. Residual (plastic) strains in the boundary layer reconstructed from experimental data]

Сопоставление результата с реконструированным профилем остаточных напряжений (рис. 12) показывает хорошее качественное и количественное соответствие ${ }^{1}$.

Заключение. В данной работе предпринята ревизия выражения, связывающего профиль остаточных напряжений с экспериментально определяемой зависимостью прогиба калибровочной пластины от толщины слоя, стравленного с упрочненной поверхности. С этой целью решена пространственная задача равновесия упругой пластины с пограничным слоем пластических деформаций в предположении однородности и изотропии поля деформаций в плоскости пластины и выполнения условий на свободных краях в слабой форме.

Показано, что формула Давиденкова-Биргера вытекает из решения упомянутой выше задачи. Также получено явное выражение, связывающее профиль пластических деформаций с экспериментально определяемой зависимостью прогиба калибровочной пластины от толщины слоя, стравленного с упрочненной поверхности. Эти данные позволяют естественным образом учесть остаточные напряжения в численной реализации задачи посредством аналогии пластических и термоупругих деформаций. Для апробации результатов были выполнены натурный и численный эксперименты и показано соответствие одного другому. При этом реализована процедура сглаживания данных лабораторных исследований, позволяющая учесть погрешность измерений.

Результаты имеют общность применения к широкому классу задач для упругих тел с упрочняющими покрытиями, а также определенную методическую ценность в усовершенствовании основ экспериментального исследования полей остаточных напряжений и пластических деформаций для разнооб-

\footnotetext{
${ }^{1}$ На рис. 13 напряжения, рассчитанные для плоского деформированного состояния, умножены на $1-\nu^{2}$ согласно $(21),(20)$ для соответствия плоскому напряженному состоянию.
} 
разных способов поверхностно-упрочняющей обработки и данных, полученных различными способами экспериментальной верификации состояния поверхностного слоя. Результаты предполагается использовать для разработки методики учета пограничного слоя остаточных напряжений при численных расчетах ресурса деталей машин стандартными пакетами прикладных вычислений.

Конкурирующие интересы. Заявляем, что в отношении авторства и публикации этой статьи конфликта интересов не имеем.

Авторский вклад и ответственность. Все авторы принимали участие в разработке концепции статьи и в написании рукописи. Авторы несут полную ответственность за предоставление окончательной рукописи в печать. Окончательная версия рукописи была одобрена всеми авторами.

Финансирование. Работа поддержана Российским фондом фундаментальных исследований (проект № 17-08-01085_а).

\section{Библиографический список}

1. Kopp R., Schulz J. Flexible sheet forming technology by double-sided simultaneous shot peen forming // CIRP Annals, 2002. vol.51, no.1. pp. 195-198. doi: 10.1016/S0007-8506(07) 61498-X.

2. Dounde A. A., Seemikeri C. Y., Tanpure P. R. Study of shot peening process and their effect on surface properties: A Review// International Journal of Engineering, Business and Enterprise Applications (IJEBEA), 2015. vol. 2, no. 12. pp. 104-107.

3. Badreddine J., Rouhaud E., Micoulaut M., Remy S. Simulation of shot dynamics for ultrasonic shot peening: Effects of process parameters // International Journal of Mechanical Sciences, 2014. vol.82. pp. 179-190. doi: 10.1016/j.ijmecsci.2014.03.006.

4. Liu Yu, Wang L., Wang D. Finite element modeling of ultrasonic surface rolling process // Journal of Materials Processing Technology, 2011. vol.211, no. 12. pp. 2106-2113. doi: 10. 1016/j.jmatprotec.2011.07.009.

5. Han B., Ju D. Y. Compressive residual stress induced by water cavitation peening: A finite element analysis // Materials and Design, 2009. vol.30, no. 8. pp. 3325-3332. doi: 10.1016/ j. matdes. 2008.11.029.

6. Champaigne J. Shot Peening Overview, 2001. 37 pp., Retrieved from https://www . shotpeener.com/library/pdf/2001012.pdf (February 14, 2018).

7. Li J. K., Mei Y., Duo W., Renzhi W. Mechanical approach to the residual stress field induced by shot peening // Materials Science and Engineering: A, 1991. vol. 147, no. 2. pp. 167-173. doi: 10.1016/0921-5093(91)90843-C.

8. Franchim A. S., de Campos V. S., Travessa D. N., de Moura Neto C. Analytical modelling for residual stresses produced by shot peening // Materials and Design, 2009. vol. 30, no. 5. pp. 1556-1560. doi: 10.1016/j.matdes .2008.07.040.

9. Sherafatnia K., Farrahi G.H., Mahmoudi A.H., Ghasemi A. Experimental measurement and analytical determination of shot peening residual stresses considering friction and real unloading behavior // Materials Science and Engineering: A, 2016. vol. 657, no. 7. pp. 309-321. doi: $10.1016 /$ j.msea.2016.01.070.

10. Davis J., Ramulu M. A study of the residual stress induced by shot peening for an isotropic material based on Prager's yield criterion for combined stresses // Meccanica, 2015. vol. 50, no. 6. pp. 1593-1604. doi : 10.1007/s11012-015-0109-0.

11. Zimmermann M., Klemenz M., Schulze V. Literature review on shot peening simulation // International Journal of Computational Materials Science and Surface Engineering, 2010. vol. 3, no. 4. pp. 289-310. doi : 10.1504/IJCMSSE. 2010.036218.

12. Rouhaud E., Deslaef D., Lu J., Chaboche J.-L. Modeling of Residual Stress, Shot Peen- 
ing / Handbook on Residual Stress; eds. Jian Lu. Society of Experimental Mechanics, 2005. pp. 116-148.

13. Gallitelli D., Boyer V., Gelineau M., Colaitis Y., Rouhaud E., Retraint D., Kubler R., Desvignes M., Barrallier L. Simulation of shot peening: From process parameters to residual stress fields in a structure // Comptes Rendus Mécanique, 2016. vol.344, no. 4-5. pp. 355374. doi: $10.1016 / j$.crme.2016.02.006.

14. Musinski W. D., McDowell D. L. On the eigenstrain application of shot-peened residual stresses within a crystal plasticity framework: Application to Ni-base superalloy specimens // International Journal of Mechanical Sciences, 2015. vol. 100. pp. 195-208. doi : 10.1016/j. ijmecsci.2015.06.020.

15. Bhuvaraghan B., Srinivasan R.M., Maffeo B. Optimization of the fatigue strength of materials due to shot peening: A survey // IJSCS-Mechanics and Applications, 2010. vol. 2, no. 2. pp. 33-63, Retrieved from https://journals.tdl.org/ijscs/index.php/ijscs/article/ view/2338/2021 (February 14, 2018).

16. Purohit R, Verma C.S., Rana R.S., Dwivedi R., Dwivedi S. Simulation of shot peening process // Materials Today: Proceedings, 2017. vol. 4, no. 2 (Part A). pp. 1244-1251. doi: 10. 1016/j.matpr.2017.01.144.

17. Xie L., Wang Ch., Wang L., Wang Zh., Jiang Ch., Lu W., Ji V. Numerical analysis and experimental validation on residual stress distribution of titanium matrix composite after shot peening treatment// Mechanics of Materials, 2016. vol. 99. pp. 2-8. doi: 10.1016/j. mechmat.2016.05.005.

18. Jebahi M., Gakwaya A., Lévesque J., Mechri O., Ba K. Robust methodology to simulate real shot peening process using discrete-continuum coupling method// International Journal of Mechanical Sciences, 2016. vol.107. pp. 21-33. doi:10.1016/j.ijmecsci.2016.01.005.

19. Биргер И. А. Остаточные напряжения. М.: Машгиз, 1963. 232 с.

20. Павлов В. Ф., Кирпичёв В. А., Вакулюк В. С. Прогнозирование сопротивления усталости поверхностно упрочненных деталей по остаточным напряжениям. Самара: СНЦ РАН, 2012. 125 с.

21. Радченко В. П., Саушкин М. Н. Ползучесть и релаксачия остаточных напряжений в упрочнённых конструкииях. М.: Машиностроение-1, 2005. 226 с.

22. Иванов С. И. К определению остаточных напряжений в цилиндре методом колец и полосок / Остаточные напряжения, Вып. 53. Куйбышев: КуАИ, 1971. С. 32-42.

23. Саушкин М. Н., Радченко В. П., Павлов В. Ф. Метод расчета полей остаточных напряжений и пластических деформаций в цилиндрических образцах с учетом анизотропии процесса поверхностного // ПМТФ, 2011. Т. 52, № 2. С. 173-182.

24. Захарова Т. П., Розанов М. А., Теплова С. В. Влияние условий эксплуатации на релаксацию остаточных напряжений сжатия в наклепанных пазах хвостовиков лопаток ТВД из жаропрочных монокристаллических никелевых сплавов // Вестник Уфимского государственного авиационного технического университета, 2015. Т. 19, № 3. С. 21-27.

25. Сазанов В. П., Кирпичев В. А., Вакулюк В. С., Павлов В. Ф. Определение первоначальных деформаций в упрочненном слое цилиндрической детали методом конечно-элементного моделирования с использованием расчетного комплекса PATRAN/NASTRAN// Вестник Уфимского государственного авиационного технического университета, 2015. Т. 19, № 2. С. 35-40.

26. Сазанов В. П., Семенова О. Ю., Кирпичев В. А., Вакулюк В. С. Математическое моделирование первоначальных деформаций в поверхностно упрочненных деталях при выборе образца-свидетеля // Вестник Уфимского государственного авиационного технического университета, 2016. Т. 20, № 3. С. 31-37.

27. Саушкин М. Н., Радченко В. П., Куров А. Ю. Метод расчета остаточных напряжений в надрезах с полукруглым профилем в полом поверхностно упрочненном цилиндрическом образце // ПМТФ, 2013. Т. 54, № 4. С. 150-157.

28. Саушкин М. Н., Куров А. Ю. Анализ напряжённого состояния в надрезах полукруглого профиля после опережающего поверхностного пластического деформирования сплош- 
ных цилиндрических образцов // Вестн. Сам. гос. техн. ун-та. Сер. Физ.-мат. науки, 2012. № 1(26). С. 133-140. doi : 10.14498/vsgtu1039.

29. Кузнецов Н. Д., Цейтлин В. И., Волков В. И. Технологические методъ повышения надёжности деталей машин: справочник. М.: Машиностроение, 1993. 304 с.

30. Mattsen R. L., Fonda H. E. Peening Intensity Measurement: Memorandum Report S-2009C: General Motors, March, 1945. 13 pp.

31. Лурье А. И. К теории толстых плит // ПММ, 1942. Т. 6, № 2-3. С. 151-168.

32. Аксентян О. К., Ворович И. И. Напряженное состояние плиты малой толщины // ПММ, 1963. Т. 27, №6. С. 1057-1074.

33. Ворович И. И., Малкина О. С. Напряженное состояние толстой плиты // ПММ, 1967. Т. 31, № 2. С. 230-241.

34. Устинов Ю. А. О структуре погранслоя в слоистых плитах// Докл. АН CCCP, 1976. Т. 229, № 2. С. 325-328.

35. Меркулова Н. С. Контроль остаточных напряжений на установке АПООН (автоматизащия прочесса определения остаточных напряжений): Производственная инструкция, НИАТ ПИ 1.4.804-84. М.: НИАТ, 1985. 50 с.

36. Келлер И. Э. Тензорное исчисление. Спб.: Лань, 2012. 176 с.

37. Koiter W. T. General theorems of elastic-plastic solids / Progress in Solid Mechanics. vol. 1; ed. J. N. Sneddon, R. Hill. Amsterdam: North Holland, 1960. pp. 167-221.

38. Zarka J., Frelat J., Inglebert G., Kasmai-Navidi P. A New Approach in Inelastic Analysis of Structures. Gif-sur-Yvette, France: CADLM, 1990. 300 pp.

39. Биргер И. А. Круглые пластинки и оболочки вращения. М.: Оборонгиз, 1961.368 с. 


\title{
MSC: $74 \mathrm{C05}, 74 \mathrm{~K} 20$
}

\section{On the reconstruction of residual stresses and strains of a plate after shot peening}

\author{
I. E. Keller ${ }^{1,2}$, V. N. Trofimov ${ }^{1}, A . V$. Vladykin ${ }^{3}$, \\ V. V. Plusnin ${ }^{3}$, D. S. Petukhov ${ }^{1,2}$, I. V. Vindokurov ${ }^{1}$ \\ 1 Perm National Research Polytechnic University, \\ 29, Komsomolsky prospekt, Perm, 614990, Russian Federation. \\ 2 Institute of Continuous Media Mechanics UB RAS, \\ 1, Academician Korolev Street, Perm, 614013, Russian Federation. \\ 3 Perm Engine Company OJSC, \\ 93, Komsomolsky prospekt, Perm, 614010, Russian Federation.
}

\begin{abstract}
The subject of this research is a mathematical description of the shape and the stress-strain state of a steel plate subjected to unilateral shot peening, its experimental verification and application of the results for verification of methods for reconstruction of residual stress and strain fields according to experimental data. Such plate is used in manufacturing as a calibrating sample to determine of shot peening duration required for formation of proper compressive tangential stress in the surface layer of the processed product. The method of calibration is convenient and widely applied in different technologies of surface hardening. In that case the source of the residual stresses is plastic strains in surface layer produced by shot peening. For the statement of the problem a plastic strain tensor field is defined up to an arbitrary function. The shape and the stress-strain state of an elastic plate with the surface layer of plastic strains were calculated numerically. The qualitative behavior of numerical solution allowed us to accept the set of hypotheses to find an analytical solution of the spatial problem of elasticity theory and to weaken the boundary conditions. The exact solution has been found analytically. Within the framework of the plane stress state along the thickness and transverse directions, the result exactly corresponds
\end{abstract}

\section{Research Article}

(우 (i) The content is published under the terms of the Creative Commons Attribution 4.0 International License (http://creativecommons.org/licenses/by/4.0/)

Please cite this article in press as:

Keller I. E., Trofimov V. N., Vladykin A. V., Plusnin V. V., Petukhov D.S., Vindokurov I. V. On the reconstruction of residual stresses and strains of a plate after shot peening, Vestn. Samar. Gos. Tekhn. Univ., Ser. Fiz.-Mat. Nauki [J. Samara State Tech. Univ., Ser. Phys. Math. Sci.], 2018, vol. 22, no. 1, pp. 40-64. doi: 10.14498/vsgtu1602 (In Russian).

\section{Authors' Details:}

Ilya E. Keller (10 http://orcid.org/0000-0001-9914-8870

Dr. Phys. \& Math. Sci.; Associate Professor; Professor, Dept. of Dynamics and Strength of Machines $^{1}$; Researcher, Lab. of Nonlinear Mechanics of Deformable Solids ${ }^{2}$; e-mail: kie@icmm.ru Victor N. Trofimov (D) http://orcid.org/0000-0001-6655-0257

Dr. Techn. Sci.; Associate Professor; Professor; Dept. of Dynamics and Strength of Machines; e-mail: tvn_perm@mail.ru

Aleksey V. Vladykin; Cand. Techn. Sci.; Deputy Chief; Center for Progressive Technologies; e-mail: vladykin-av@pmz.ru 
to the Davidenkov-Birger formula connected the tangential residual stress distribution on depth with the function of deflections. An explicit formula for the dependence of the residual (plastic) deformation on the thickness coordinate is obtained. Sources of errors of the received expressions and methods of their correction are analyzed. An experiment has been carried out on the one-sided shot peening of calibration plate made of hardened $65 \mathrm{G}$ steel, for which the layer-by-layer etching of the treated surface and the measurement of the flexure of the plate were made (by Davidenkov method). The profiles of residual stresses and strains were reconstructed numerically with reasonable accuracy using the obtained experimental data. The result is applicable to a wide class of problems for elastic bodies with hardened surface layers. It may serve as a base for experimental research of such problems, help to formulate hypotheses and test them by experiment, help to study relation between physical fields in asymptotic case, help to verify applicability of different ways to account residual stresses in numerical solution. The solution found can be used for verification of stress and displacement fields in different cases of preliminarily stressed shell elements in engineering software for calculation of fatigue endurance of different machine parts with hardened surface layer. It also seems to be a reference for the study of surface-hardened bodies with curved free boundary, to which most of the practically important tasks are reduced.

Keywords: residual stresses, boundary layer, shot peening, surface treatment, experiment, calculation, exact solution, reconstruction.

Received: $16^{\text {th }}$ January, $2018 /$ Revised: $27^{\text {th }}$ February, 2018/ Accepted: $12^{\text {th }}$ March, $2018 /$ First online: $29^{\text {th }}$ March, 2018

Competing interests. We declare that we have no conflicts of interests with the authorship and publication of this article.

Authors' contributions and responsibilities. Each author has participated in the article concept development and in the manuscript writing. The authors are absolutely responsible for submitting the final manuscript in print. Each author has approved the final version of manuscript.

Funding. This work was supported by the Russian Foundation for Basic Research (project no. 17-08-01085_a).

Viktor V. Plyusnin; Head; Dept. of Physical and Mechanical Research; e-mail: dolgih-nv@pmz.ru

Dmitriy S. Petukhov (D) http://orcid.org/0000-0002-6996-3580

Assistant, Dept. of Dynamics and Strength of Machines ${ }^{1}$; Postgraduate Student, Lab. of Nonlinear Mechanics of Deformable Solids'; e-mail: petuhovds@mail.ru

Ilya V. Vindokurov (1) http://orcid.org/0000-0002-1885-0404

Student; Dept. of Dynamics and Strength of Machines; e-mail: dpmm17@mail.ru 


\section{References}

1. Kopp R., Schulz J. Flexible sheet forming technology by double-sided simultaneous shot peen forming, CIRP Annals, 2002, vol.51, no.1, pp. 195-198. doi:10.1016/S0007-8506(07) 61498-X.

2. Dounde A. A., Seemikeri C. Y., Tanpure P. R. Study of shot peening process and their effect on surface properties: A Review, International Journal of Engineering, Business and Enterprise Applications (IJEBEA), 2015, vol. 2, no. 12, pp. 104-107.

3. Badreddine J., Rouhaud E., Micoulaut M., Remy S. Simulation of shot dynamics for ultrasonic shot peening: Effects of process parameters, International Journal of Mechanical Sciences, 2014, vol.82, pp. 179-190. doi:10.1016/j.ijmecsci.2014.03.006.

4. Liu Yu, Wang L., Wang D. Finite element modeling of ultrasonic surface rolling process, Journal of Materials Processing Technology, 2011, vol.211, no. 12, pp. 2106-2113. doi: 10. 1016/j.jmatprotec.2011.07.009.

5. Han B., Ju D. Y. Compressive residual stress induced by water cavitation peening: A finite element analysis, Materials and Design, 2009, vol.30, no. 8, pp. 3325-3332. doi: 10.1016/ j.matdes.2008.11.029.

6. Champaigne J. Shot Peening Overview, 2001, 37 pp., Retrieved from https://www. shotpeener.com/library/pdf/2001012.pdf (February 14, 2018).

7. Li J. K., Mei Y., Duo W., Renzhi W. Mechanical approach to the residual stress field induced by shot peening, Materials Science and Engineering: A, 1991, vol.147, no. 2, pp. 167-173. doi: 10.1016/0921-5093(91)90843-C.

8. Franchim A. S., de Campos V. S., Travessa D. N., de Moura Neto C. Analytical modelling for residual stresses produced by shot peening, Materials and Design, 2009, vol. 30, no. 5, pp. 1556-1560. doi: 10.1016/j.matdes.2008.07.040.

9. Sherafatnia K., Farrahi G.H., Mahmoudi A.H., Ghasemi A. Experimental measurement and analytical determination of shot peening residual stresses considering friction and real unloading behavior, Materials Science and Engineering: A, 2016, vol. 657, no. 7, pp. 309-321. doi: $10.1016 /$ j.msea.2016.01.070.

10. Davis J., Ramulu M. A study of the residual stress induced by shot peening for an isotropic material based on Prager's yield criterion for combined stresses, Meccanica, 2015, vol. 50, no. 6, pp. 1593-1604. doi : 10.1007/s11012-015-0109-0.

11. Zimmermann M., Klemenz M., Schulze V. Literature review on shot peening simulation, International Journal of Computational Materials Science and Surface Engineering, 2010, vol. 3, no. 4, pp. 289-310. doi: 10.1504/IJCMSSE.2010.036218.

12. Rouhaud E., Deslaef D., Lu J., Chaboche J.-L. Modeling of Residual Stress, Shot Peening, In: Handbook on Residual Stress; eds. Jian Lu. Society of Experimental Mechanics, 2005, pp. 116-148.

13. Gallitelli D., Boyer V., Gelineau M., Colaitis Y., Rouhaud E., Retraint D., Kubler R., Desvignes M., Barrallier L. Simulation of shot peening: From process parameters to residual stress fields in a structure, Comptes Rendus Mécanique, 2016, vol. 344, no. 4-5, pp. 355-374. doi: 10.1016/j.crme.2016.02.006.

14. Musinski W. D., McDowell D. L. On the eigenstrain application of shot-peened residual stresses within a crystal plasticity framework: Application to Ni-base superalloy specimens, International Journal of Mechanical Sciences, 2015, vol. 100, pp. 195-208. doi : 10.1016/j . ijmecsci.2015.06.020.

15. Bhuvaraghan B., Srinivasan R.M., Maffeo B. Optimization of the fatigue strength of materials due to shot peening: A survey, IJSCS-Mechanics and Applications, 2010, vol. 2, no. 2, pp. 33-63, Retrieved from https://journals.tdl.org/ijscs/index.php/ijscs/article/ view/2338/2021 (February 14, 2018).

16. Purohit R, Verma C.S., Rana R.S., Dwivedi R., Dwivedi S. Simulation of shot peening process, Materials Today: Proceedings, 2017, vol. 4, no. 2 (Part A), pp. 1244-1251. doi: 10. 1016/j.matpr.2017.01.144. 
17. Xie L., Wang Ch., Wang L., Wang Zh., Jiang Ch., Lu W., Ji V. Numerical analysis and experimental validation on residual stress distribution of titanium matrix composite after shot peening treatment, Mechanics of Materials, 2016, vol.99, pp. 2-8. doi: 10.1016/j. mechmat.2016.05.005.

18. Jebahi M., Gakwaya A., Lévesque J., Mechri O., Ba K. Robust methodology to simulate real shot peening process using discrete-continuum coupling method, International Journal of Mechanical Sciences, 2016, vol.107, pp. 21-33. doi:10.1016/j.ijmecsci.2016.01.005.

19. Birger I. A. Ostatochnye napriazheniia [Residual Stresses]. Moscow, Mashgiz, 1963, 232 pp. (In Russian)

20. Pavlov V. F., Kirpichev V. A., Vakulyuk V. S. Prognozirovanie soprotivleniia ustalosti poverkhnostno uprochnennykh detalei po ostatochnym napriazheniiam [Prediction of fatigue resistance of surface-hardened parts by residual stresses]. Samara, Samara Scientific Center of RAS, 2012, 125 pp. (In Russian)

21. Radchenko V. P., Saushkin M. N. Polzuchest' i relaksatsiia ostatochnykh napriazhenii v uprochnennykh konstruktsiiakh [Creep and Relaxation of Residual Stresses in Hardened Structures]. Moscow, Mashinostroenie-1, 2005, 226 pp. (In Russian)

22. Ivanov S. I. The Way to Determine the Residual Stresses in a Cylinder by Using the Rings and Stripes Method, In: Ostatochnye napriazheniia [Residual Stresses], Issue 53. Kuibyshev, KuAI, 1971, pp. 32-42 (In Russian).

23. Saushkin M. N., Radchenko V. P., Pavlov V. F. Method of calculating the fields of residual stresses and plastic strains in cylindrical specimens with allowance for surface hardening anisotropy, J. Appl. Mech. Tech. Phys., 2011, vol.52, no.2, pp. 303-310. doi:10.1134/ S0021894411020180.

24. Zakharova T. P., Rozanov M. A., Teplova S. V. Influence of operation conditions at the residual stress relaxation in turbine root slots of HPT, made of monocrystal nickel super alloys, Vestnik UGATU, 2015, vol. 19, no. 3, pp. 21-27 (In Russian).

25. Sazanov V. P., Kirpichev V. A., Vakulyuk V. S., Pavlov V. F. The definition of initial deformations in the cylindrical parts surface layer by finite elements modeling method using PATRAN/NASTRAN program complex, Vestnik UGATU, 2015, vol. 19, no. 2, pp. 35-40 (In Russian).

26. Sazanov V. P., Semenova O. Yu., Kirpichev V. A., Vakulyuk V. S. Mathematical modeling of initial deformations in surface hardened parts for the choice of a reference specimen, Vestnik UGATU, 2016, vol. 20, no. 3, pp. 31-37 (In Russian).

27. Saushkin M. N., Radchenko V. P., Kurov A. Yu. Method of calculating residual stresses in semicircular notches in a surface hardened hollow cylindrical specimen, J. Appl. Mech. Tech. Phys., 2013, vol. 54, no. 4, pp. 644-650. doi: 10.1134/S0021894413040159.

28. Saushkin M. N., Kurov A. Yu. Analysis of stress state in semicircular profile notches after preliminary surface plastic deformation of solid cylindrical specimens, Vestn. Samar. Gos. Tekhn. Univ., Ser. Fiz.-Mat. Nauki [J. Samara State Tech. Univ., Ser. Phys. Math. Sci.], 2012, no. 1(26), pp. 133-140 (In Russian). doi : 10.14498/vsgtu1039.

29. Kuznetsov N. D., Tseitlin V. I., Volkov V. I. Tekhnologicheskie metody povysheniia nadezhnosti detalei mashin [Technological Techniques for Improving Reliability of Machine Parts]. Moscow, Mashinostroenie, 1993, 304 pp. (In Russian)

30. Mattsen R. L., Fonda H. E. Peening Intensity Measurement, Memorandum Report S-2009C, General Motors, March, 1945, 13 pp.

31. Lurie A. I. To the theory of thick plates, Prikl. Mat. Mekh., 1942, vol. 6, no. 2-3, pp. 151-168 (In Rusiian).

32. Aksentian O. K., Vorovich I. I. The state of stress in a thin plate, J. Appl. Math. Mech., 1963, vol. 27, no. 6, pp. 1621-1643. doi : 10.1016/0021-8928(63)90129-1.

33. Vorovich I. I., Malkina O. S. The state op stress in a thick plate, J. Appl. Math. Mech., 1967, vol.31, no. 2, pp. 252-264. doi: 10.1016/0021-8928(67)90150-5.

34. Ustinov Yu. A. The structure of the boundary layer in laminated plates, Dokl. Akad. Nauk SSSR, 1976, vol. 229, no. 2, pp. 325-328 (In Russian). 
35. Merkulova N. S. Monitoring of residual stresses at the installation for the Automation of the Residual Stresses Determination Process (ARSDP), The procedures manual, NIAT PI 1.4.804-84. Moscow, National Institute of Aviation Technologies, 1985, 50 pp. (In Russian)

36. Keller I. E. Tenzornoe ischislenie [Tensor Calculus]. St. Petersburg, Lan', 2012, 176 pp. (In Russian)

37. Koiter W. T. General theorems of elastic-plastic solids, In: Progress in Solid Mechanics, vol. 1; ed. J. N. Sneddon, R. Hill. Amsterdam, North Holland, 1960, pp. 167-221.

38. Zarka J., Frelat J., Inglebert G., Kasmai-Navidi P. A New Approach in Inelastic Analysis of Structures. Gif-sur-Yvette, France, CADLM, 1990, 300 pp.

39. Birger I. A. Kruglye plastinki i obolochki vrashcheniia [Round Plates and Shells of Revolution]. Moscow, Oborongiz, 1961, 368 pp. (In Russian) 